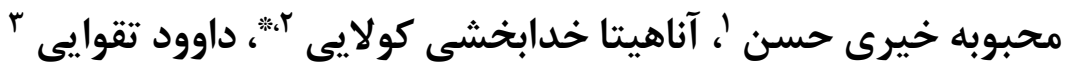

' كارشناس ارشد، كروه روان شناسى، دانشكاه آزاد اسلامى، واحد اراك، اراك، ايران ط استاديار، كروه مشاوره و روان شناسى، دانشكده علوم انسانى، دانشكاه خاتم، تهران، ايران " استاديار، كروه روان شناسى، دانشخاه آزاد اسلامى، واحد اراك، اراك، ايران " نويسنده مسئول: آناهيتا خدابخشى كولايى، استاديار، گروه مشاوره و روان شناسى، دانشكده علوم انسانى، دانشعاه خاتم، آنان.

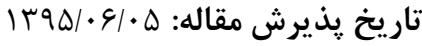
a.khodabakhshid@khatam.ac.ir : تهران، ايران. ايميل جكيده

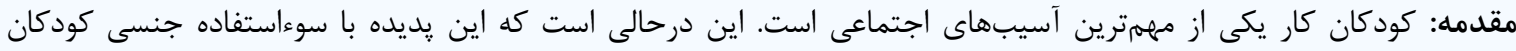

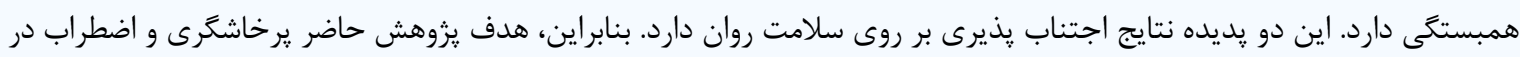

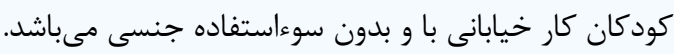

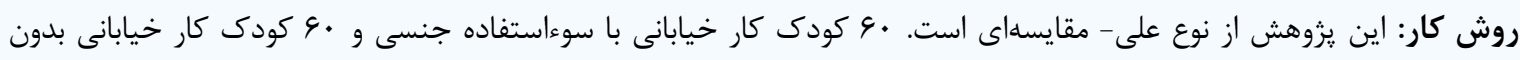

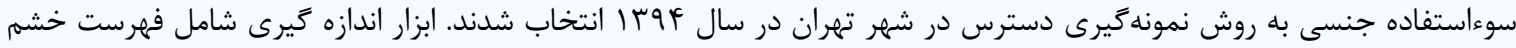

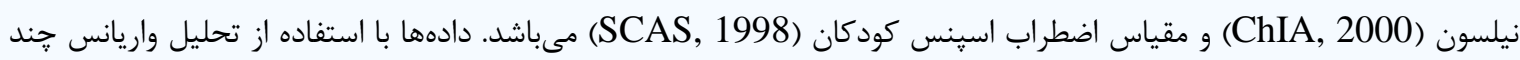
متغيرى (MANOVA) مورد تحليل قرار كرفت.

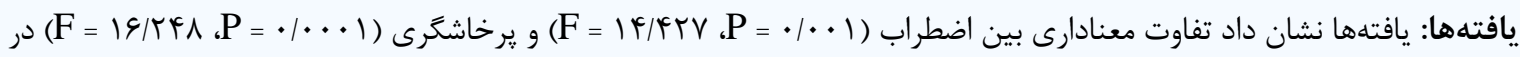

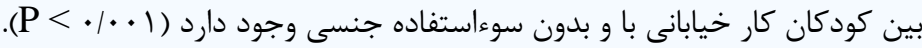

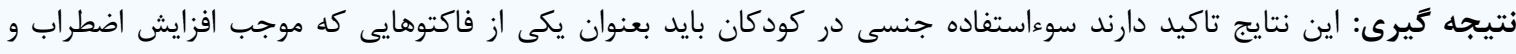

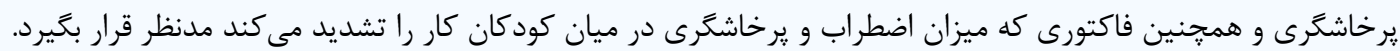

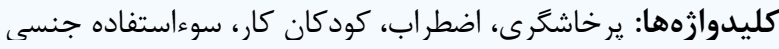

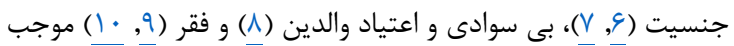

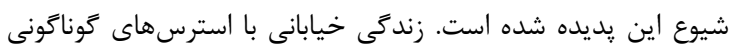

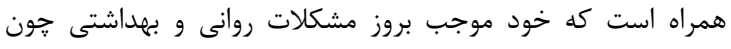

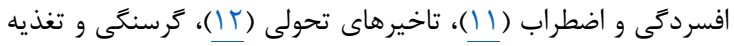

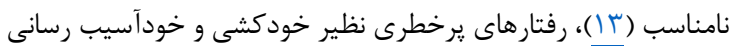

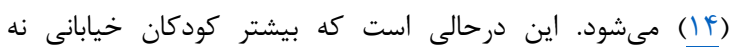

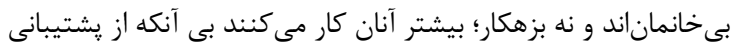

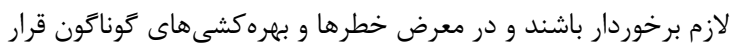

دارند (1) (1). يكى از عمدهترين خطراتى كه كودكان كار را تهديد مى كند سوءاستفاده ) ارها

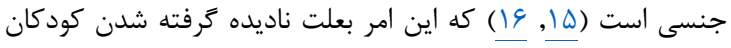

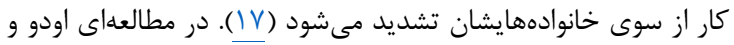
همكاران بر روى عاس كودى كار دختر در نيجريه نشان دادند
كودكان كار در يك نحاه كلى در آستانه آسيبذيذيرى و رفتارهاى

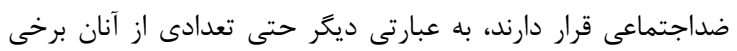

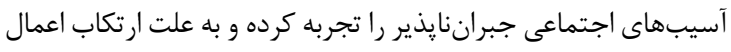

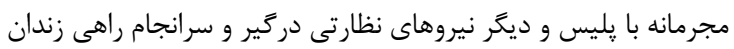

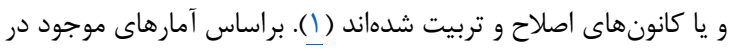

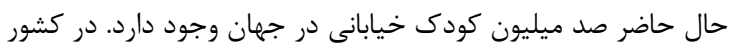

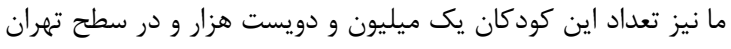

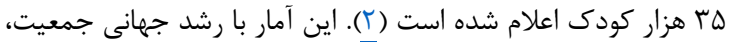

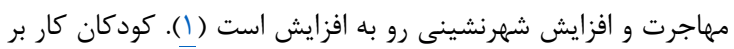

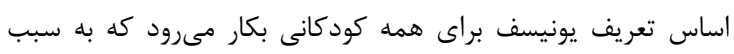

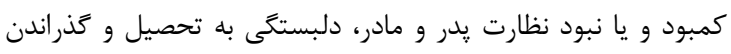

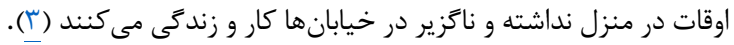

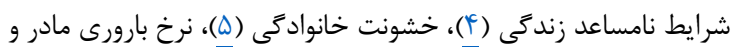


خشم و مهارتهاى اجتماعى در كودكان سنين 9 تا 1 سال تهيه شده

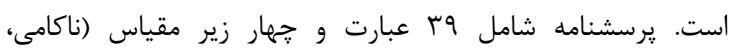

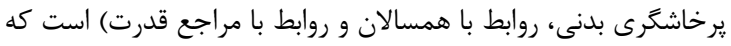

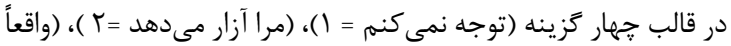

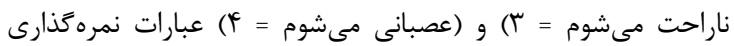

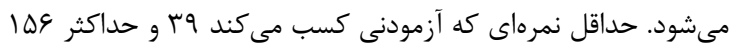

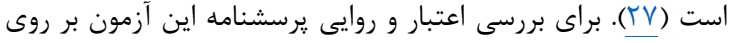

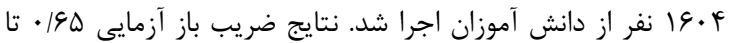

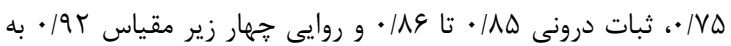

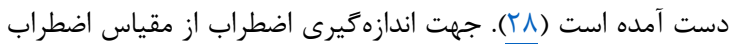

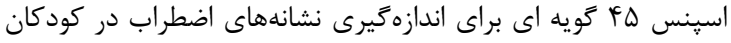

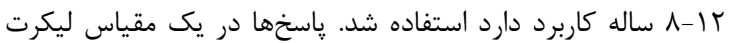

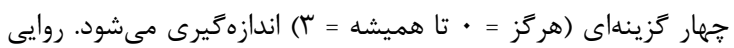

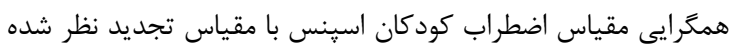

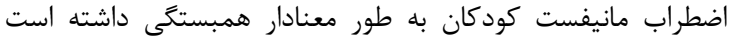

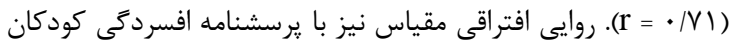

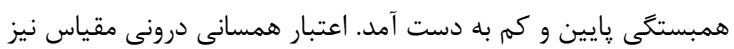

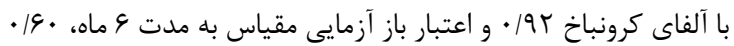
بدست آمد (99) اين يرسشنامه توسط موسوى و همكاران ترجمه شده

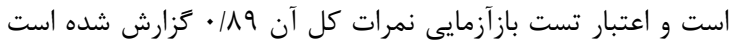

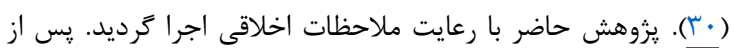

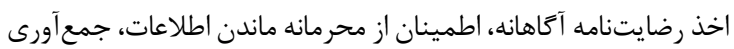

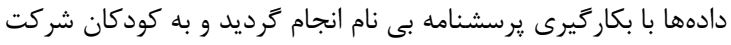

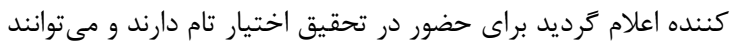

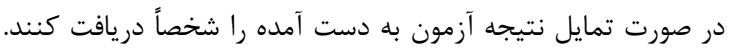

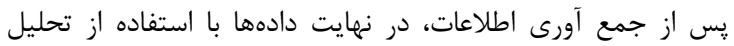

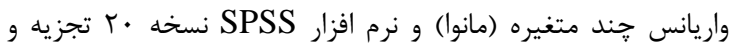

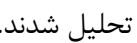

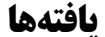

نتايج بدست آمده نشان داد س/F/N د درصد ازكودكان ترك تحصيل كرده

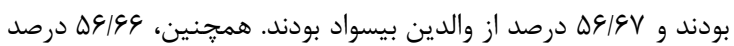
از קدران داراى شغل آزاد و و

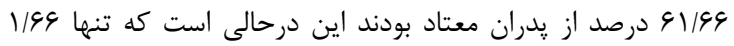

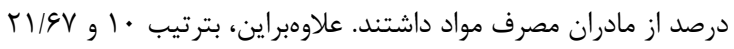

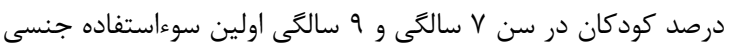

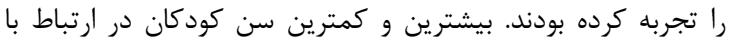

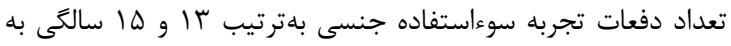

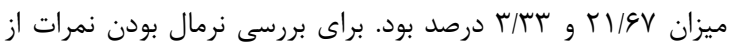

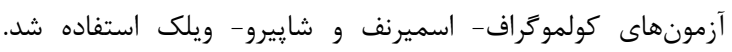

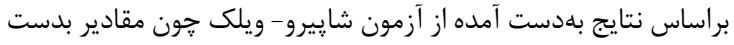

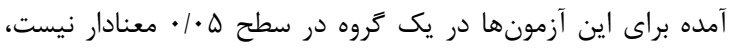

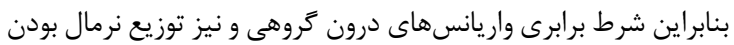

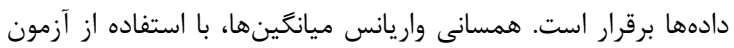

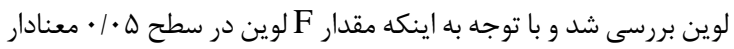

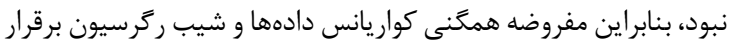
مىباشد.
درصد اين كودكان تجربه سوءاستفاده جنسى را داشتهاند (ع) (آزار

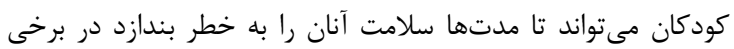

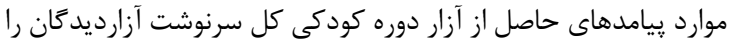

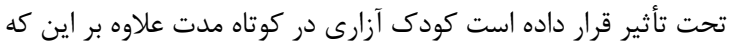

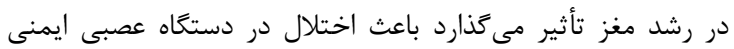

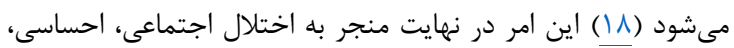

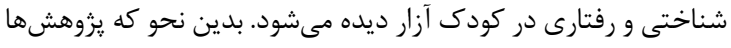

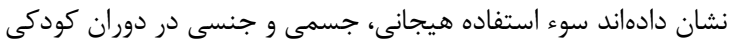

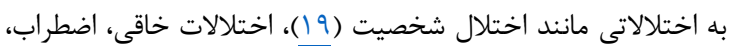

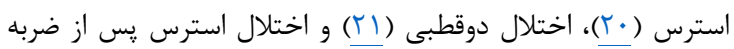

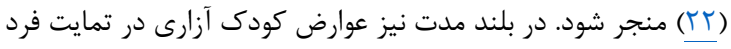

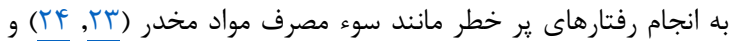

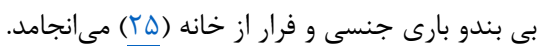

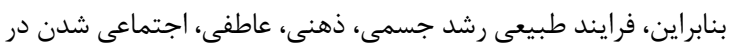

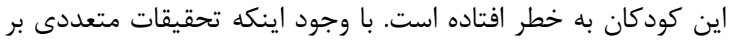

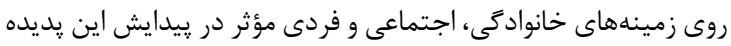

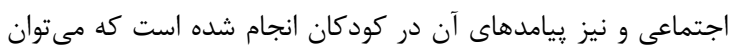

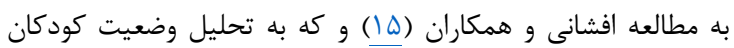

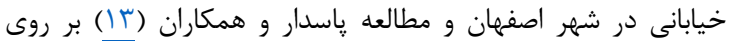

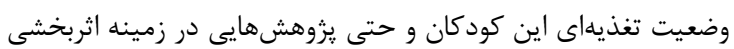

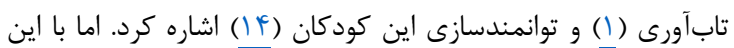

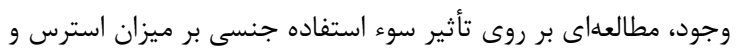

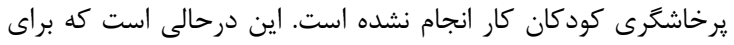

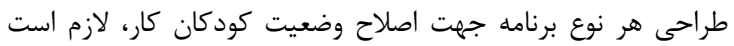

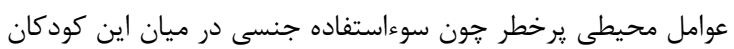

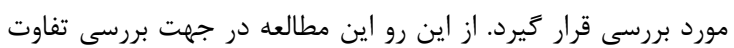

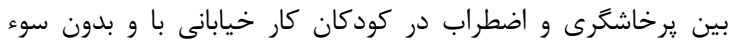

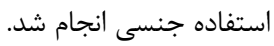

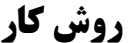

تحقيق حاضر از نوع على - مقايسهاى است. جامعه آمارى اين يزوهش

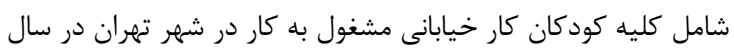

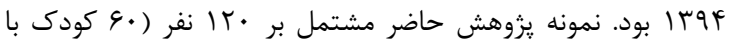

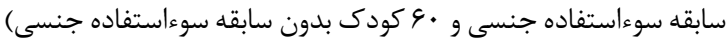

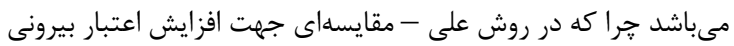

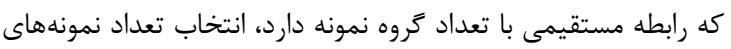

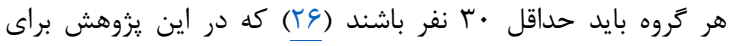

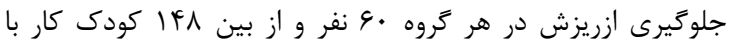

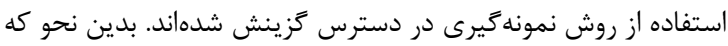

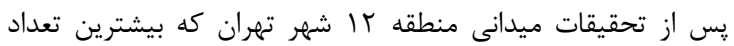

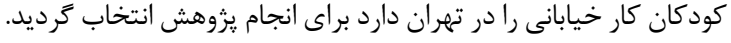

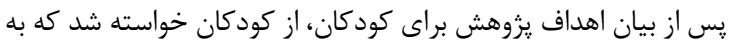

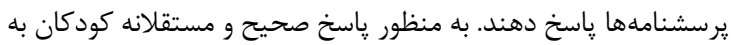

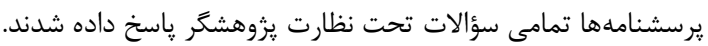

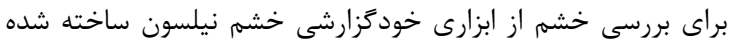

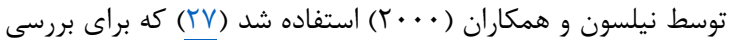
موقعيتهاى مختلفى كه موجب بروز خشم و همجنين ميزان شدت 
تفاوت معنادارى وجود دارد. طبق جدول r، دادههاى بدست آمده از

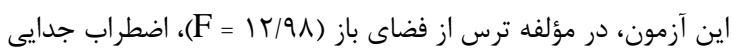
(F=V/FY)

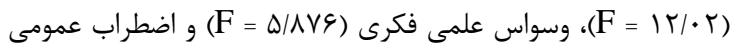

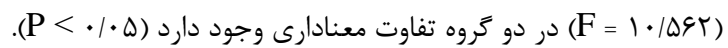

همجنين، نتايج جدول ا، نشان مىدهد كه بين در مؤلفه ناكامى (F)

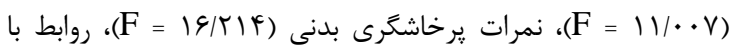

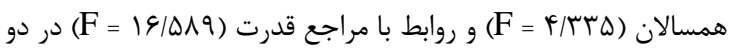

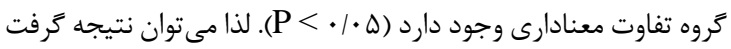

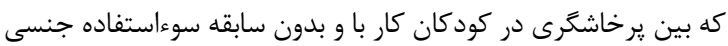

\begin{tabular}{|c|c|c|c|c|c|c|}
\hline مجذور اتا & سطح معنادارى & $\mathbf{F}$ & ميانگين مجذورات & درجات آزادى & مجموع مجذورات & منابع تغييرات \\
\hline & & & & & & اثر كروه \\
\hline$\cdot / \cdot \wedge \Delta$ & $\cdot 1 \cdot \cdots 1$ & $11 / \cdots v$ & $\Delta T r / \Delta \wedge \Delta$ & 1 & $\Delta T Y / \Delta \wedge \Delta$ & 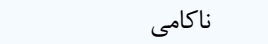 \\
\hline$\cdot|| T \mid$ & $\cdot 1 \cdot \cdots 1$ & $|9 / \pi| f$ & & 1 & & يرخاشگَرى بدنى \\
\hline$\cdot / \cdot r \Delta$ & $\cdot \mid \cdot \boldsymbol{r}$ & ( & $|f r| \cdots \wedge$ & 1 & $|f r| \cdots \wedge$ & روابط با همسالان \\
\hline \multirow[t]{2}{*}{ (Ir } & $\cdot 1 \cdot \cdots \cdot 1$ & $19 / 091$ & $V F \cdot / \cdot r r$ & 1 & $V F \cdot 1 \cdot r r$ & روابط با مراجع قدرت \\
\hline & & & & & & خطا \\
\hline- & - & - & FV/FVG & 111 & $\Delta G \cdot T / I V$ & 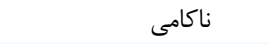 \\
\hline- & - & - & $r \Delta / r \Lambda I$ & 111 & $F \mid V T / I S V$ & يرخاشگرى بدنى \\
\hline - & - & - & rT/99l & 111 & 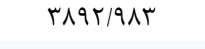 & روابط با همسالان \\
\hline \multirow[t]{2}{*}{-} & - & - & $F F / \Delta \wedge G$ & 111 & $\Delta r \varepsilon I / 19 V$ & روابط با مراجع قدرت \\
\hline & & & & & & كل \\
\hline - & - & - & - & $M$ & $1 \cdot 94 \cdot T / V 19$ & 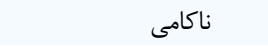 \\
\hline - & - & - & - & $\pi$ & $1 \cdot V \vee \Delta F / \cdots$ & يرخاشگرى بدنى \\
\hline - & - & - & - & $\pi$ & $\wedge \notin 9 \vee \backslash / \cdots$ & روابط با همسالان \\
\hline- & - & - & - & $1 T \cdot$ & $1 \cdot \Delta \mu F / \cdots$ & روابط با مراجع قدرت \\
\hline
\end{tabular}

جدول r: خلاصه نتايج آزمون تحليل كواريانس خند متغيره در مورد مقايسه اضطراب در كودكان كار باو بدون سابقه سوء استفادى جنسى مئى

\begin{tabular}{|c|c|c|c|c|c|c|}
\hline \multirow[t]{2}{*}{ مجذور اتا } & سطح معنادارى & $\mathbf{F}$ & ميانكين مجذورات & درجات آزادى & مجموع مجذورات & منابع تغييرات \\
\hline & & & & & & اثر تروه \\
\hline .1 .99 & $\cdot|\cdots|$ & $\mid r / 9 \wedge \Delta$ & $r r \cdot 19 V T$ & 1 & $r r \cdot 19 V T$ & ترس از فضاى باز \\
\hline$\cdot 1 \cdot \Delta q$ & $\cdot \cdot \cdot v$ & $V / F F A$ & $\| V / F \mid F$ & 1 & $\|V / F\|^{f}$ & اضطراب جدايى \\
\hline$\cdot / 1 Y F$ & $\cdot \mid \cdots \cdot 1$ & $\mid N / r \wedge 9$ & TFY/AIG & 1 & TEF/AIS & ترس از آسيب فيزيكى \\
\hline . &.$/ .1$ & $\mid r / \cdot r q$ & $1 . r / 9 V F$ & 1 & $1 . r / q V F$ & ترس اجتماعى \\
\hline $.1 \cdot \psi v$ &.$/ .1 \mathrm{~V}$ & D/AVG & GK/TSD & 1 & GK/TSD & وسواس علمى فكرى \\
\hline \multirow[t]{2}{*}{ 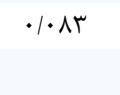 } & . $/ . r$ & $1 \cdot / \Delta G T$ & 1. & 1 & 1. F/DTr & اضطراب عمومى \\
\hline & & & & & & خطا \\
\hline- & - & - & $r Q / \& \& V$ & 111 & $r \cdot \cdot \Delta / \cdot \Delta \varphi$ & ترس از فضاى باز \\
\hline- & - & - & $10 / V G \varphi$ & 111 & ING./rTF & اضطراب جدايى \\
\hline- & - & - & $\mid r / r \Lambda \Lambda$ & 111 & $|\Delta V Q / A T|$ & ترس از آسيب فيزيكى \\
\hline- & - & - & NGFF & 111 & $1.19 / 999$ & ترس اجتماعى \\
\hline- & - & - & 1.VNAr & 111 & ITVT/K.9 & وسواس علمى فكرى \\
\hline \multirow[t]{2}{*}{-} & - & - & $9 / 197$ & 111 & MTHAN/AT & اضطراب عمومى \\
\hline & & & & & & كل \\
\hline- & - & - & - & ir. & $1.949 / 1 \%$. & ترس از فضاى باز \\
\hline- & - & - & - & Ir. & VTOV/AD. & اضطراب جدايى \\
\hline- & - & - & - & Ir. & GQDQ/VT. & ترس از آسيب فيزيكى \\
\hline- & - & - & - & Ir. & एद१९//Q. & ترس اجتماعى \\
\hline- & - & - & - & Ir. & $T M \Delta F / I T$. & وسواس علمى فكرى \\
\hline- & - & - & - & ir. & $\Delta V G Y / \cdots$ & اضطراب عمومى \\
\hline
\end{tabular}




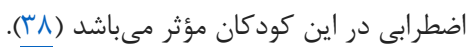

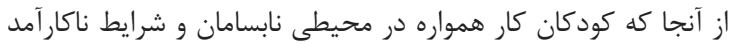

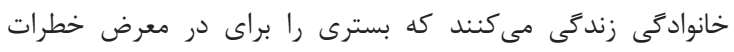

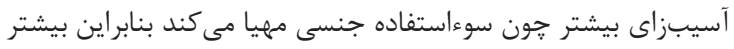

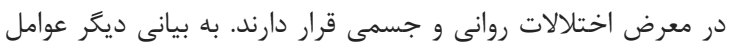

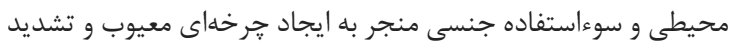

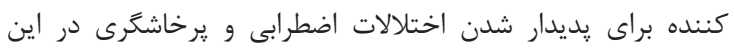

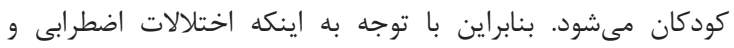

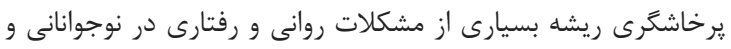

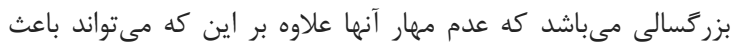

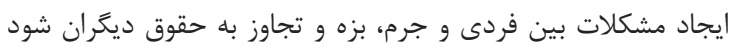

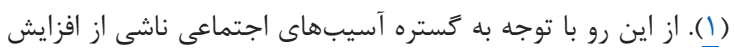

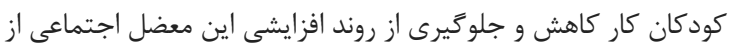

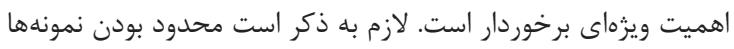

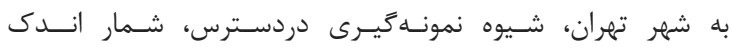

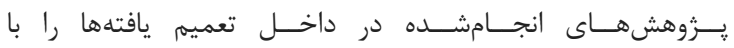

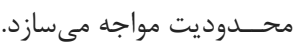

\section{نتيجه تيرى}

اتر خه با توجه به محيط و شرايط نابسامان كودكان كار ذاتاً ميزان

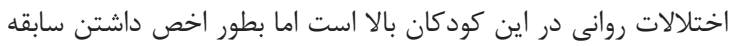

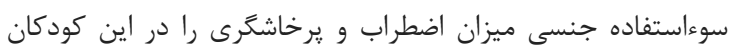

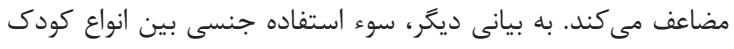

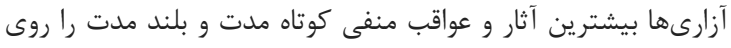

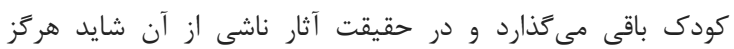

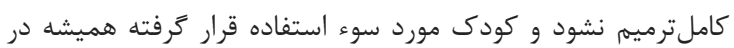

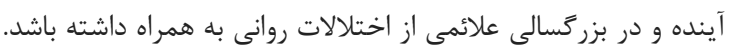

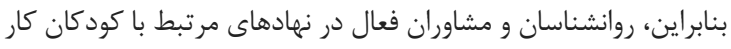

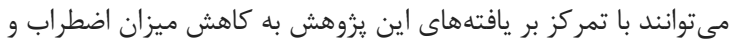

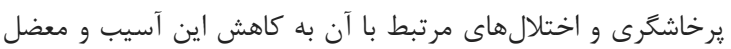
اجتماعى كمك شايانى كنند.

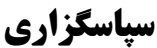

اين مقاله برَّرفته از يايان نامه كارشناسى ارشد نويسنده اول دفاع شده

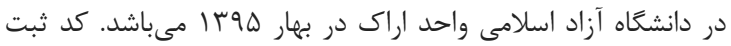

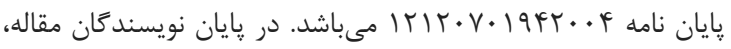

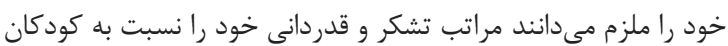

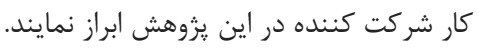

\section{References}

1. Siadat S. [Effects of social skills teaching on the selfesteem and identity dimensions of the labor children]. Educ Psychol. 2012;11(37):83-101.

2. Farzad V, Emamipur S, Vakil Ghahani F. Investigation of validity and reliability of The Child Symptom Inventory-4 (CSI-4) on labor children. J Psychol Res. 2011;3(11):47-57.
يروهش حاضر به منظور بررسى يرخاشكرى و اضطراب در كودكان كار

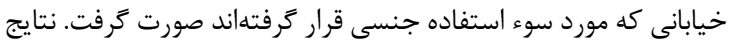

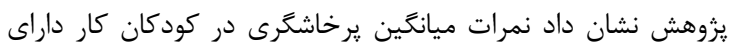

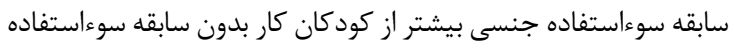

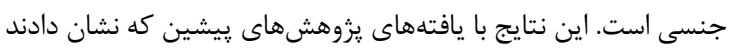

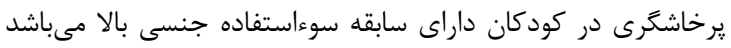

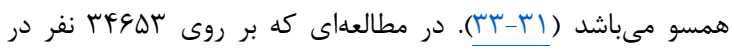

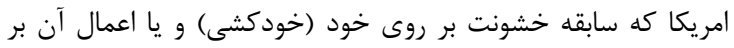

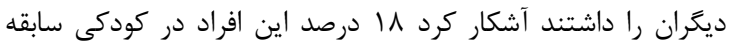

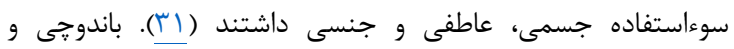

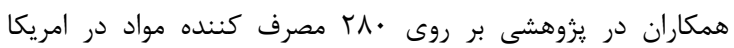

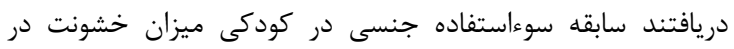

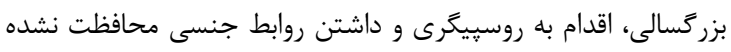

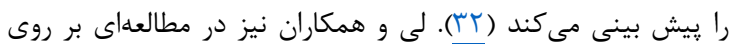

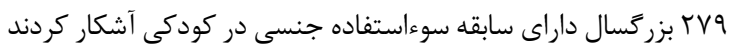

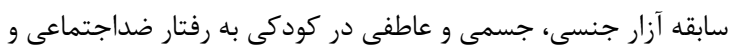

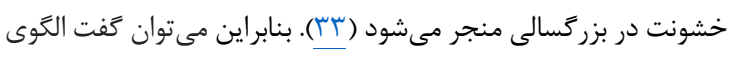

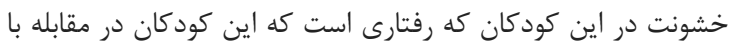

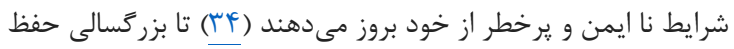

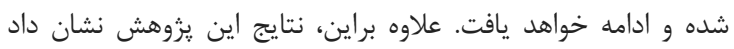

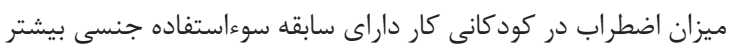

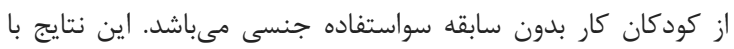

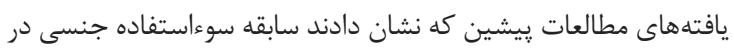

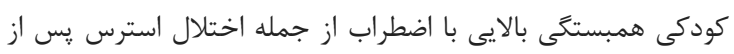

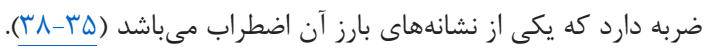

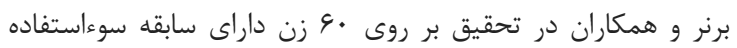

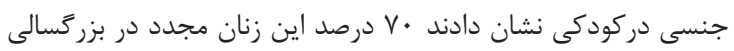

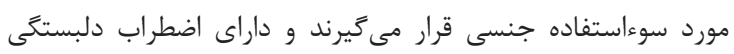

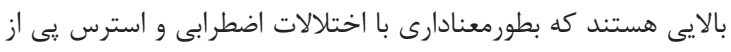

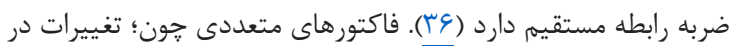

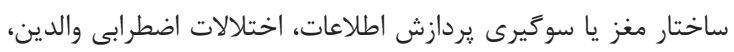

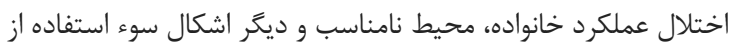

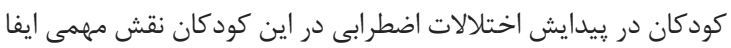

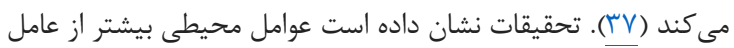
زنتيك در ايجاد اختلالات اضطرابى مؤثر هستند. تجربيات تروماتيك داني

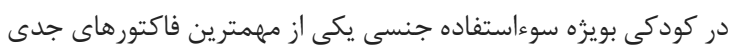

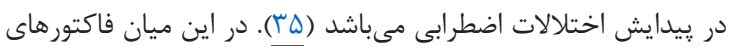

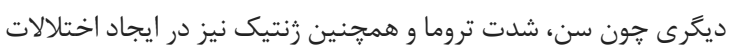

3. Dayığlu M. How sensitive are estimates of working children and child labour to definitions? A comparative analysis. New York: Unicef Statistics and Monitoring section Dopas; 2012 Contract No.: 1.

4. Rodríguez C, Sánchez F. Armed Conflict Exposure, Human Capital Investments, And Child Labor: Evidence From Colombia. Defence Peace Econom. 
2012;23(2):161-84.

DOI:

$\underline{10.1080 / 10242694.2011 .597239}$

5. Catani C, Schauer E, Elbert T, Missmahl I, Bette JP, Neuner F. War trauma, child labor, and family violence: life adversities and PTSD in a sample of school children in Kabul. J Trauma Stress. 2009;22(3):163-71. DOI: 10.1002/jts.20415 PMID: 19462436

6. Homaie Rad E, Gholampoor H, Jaafaripooyan E. Child Labor and the Influencing Factors: Evidence from less Developed Provinces of Iran. Iranian J Public Health. 2015;44(9):1244.

7. Edmonds EV, Shrestha M. You get what you pay for: Schooling incentives and child labor. J Dev Econom. 2014;111(2):196-211.

DOI: 10.1016/j.jdeveco.2014.09.005

8. Webbink E, Smits J, de Jong E. Hidden Child Labor: Determinants of Housework and Family Business Work of Children in 16 Developing Countries. World Dev. 2012;40(3):631-42. DOI: 10.1016/j.worlddev.2011.07.005

9. Bandara A, Dehejia R, Lavie-Rouse S. The Impact of Income and Non-Income Shocks on Child Labor: Evidence from a Panel Survey of Tanzania. World Dev. 2015;67(4):218-37. DOI: 10.1016/j.worlddev.2014.10.019

10. Maconachie R, Hilson G. Re-Thinking the Child Labor "Problem" in Rural sub-Saharan Africa: The Case of Sierra Leone's Half Shovels. World Dev. 2016;78(3):136-47.

10.1016/j.worlddev.2015.10.012

11. Doku PN. Depression, Delinquency and Peer Problems Among Children and Adolescents Affected by HIV/AIDS in Ghana: The Mediating Role of Child Labour. J Depression Anxiety. 2016;05(02):245-80. DOI: $10.4172 / 2167-1044.1000221$

12. Dousti M, Pourmohamadrezatajrishi M, Ghobari BB. The effectiveness of resilience training on psychological well-being of female street children with externalizing disorders. J Dev Psychol. 2014;11(41):43-54.

13. Pasdar Y, Darbandi M, Nachvak SM. Nutritional status of working children as a neglected group in Kermanshah. J Commun Health Res. 2014;3(1):12431.

14. Ayatmehr F, Khaleghi M, Mohammadkhani S, Ghiasi H. [The Efficacy of Education of Adolescent Empowerment in Prevention of Risky Behaviors in Child Labor]. Q J Soc Work. 2015;4(1):16-23.

15. Afshani SA, Askari-Nodoushan A, Heydari M, Noorian Najafabadi M. An Analysis of the StreetChildren Phenomenon in the City of Isfahan. J Appl Sci. 2013;4(2):21-5.

16. Audu B, Geidam A, Jarma H. Child labor and sexual assault among girls in Maiduguri, Nigeria. Int $\mathbf{J}$ Gynaecol Obstet. 2009;104(1):64-7. DOI: 10.1016/j.ijgo.2008.09.007 PMID: 18954870
17. Milaniak I, Widom CS. Does Child Abuse and Neglect Increase Risk for Perpetration of Violence Inside and Outside the Home? Psychol Violence. 2015;5(3):246-55. DOI: 10.1037/a0037956 PMID: 26191459

18. Weder N, Zhang H, Jensen K, Yang BZ, Simen A, Jackowski A, et al. Child abuse, depression, and methylation in genes involved with stress, neural plasticity, and brain circuitry. J Am Acad Child Adolesc Psychiatry. 2014;53(4):417-24 e5. DOI: 10.1016/j.jaac.2013.12.025 PMID: 24655651

19. Kuo JR, Khoury JE, Metcalfe R, Fitzpatrick S, Goodwill A. An examination of the relationship between childhood emotional abuse and borderline personality disorder features: the role of difficulties with emotion regulation. Child Abuse Negl. 2015;39:147-55. DOI: 10.1016/i.chiabu.2014.08.008 PMID: 25192957

20. Boroughs MS, Valentine SE, Ironson GH, Shipherd JC, Safren SA, Taylor SW, et al. Complexity of childhood sexual abuse: predictors of current posttraumatic stress disorder, mood disorders, substance use, and sexual risk behavior among adult men who have sex with men. Arch Sex Behav. 2015;44(7):1891-902. DOI: 10.1007/s10508-0150546-9 PMID: 26159863

21. Post RM, Altshuler LL, Kupka R, McElroy SL, Frye MA, Rowe M, et al. Verbal abuse, like physical and sexual abuse, in childhood is associated with an earlier onset and more difficult course of bipolar disorder. Bipolar Disord. 2015;17(3):323-30. DOI: 10.1111/bdi.12268 PMID: 25307301

22. Nemeroff CB. Paradise Lost: The Neurobiological and Clinical Consequences of Child Abuse and Neglect. Neuron. 2016;89(5):892-909. DOI: 10.1016/j.neuron.2016.01.019 PMID: 26938439

23. Banducci AN, Hoffman E, Lejuez CW, Koenen KC. The relationship between child abuse and negative outcomes among substance users: psychopathology, health, and comorbidities. Addict Behav. 2014;39(10):1522-7.

DOI: 10.1016/j.addbeh.2014.05.023 PMID: 24976457

24. Ogai Y, Senoo E, Gardner FC, Haraguchi A, Saito T, Morita N, et al. Association between experience of child abuse and severity of drug addiction measured by the Addiction Severity Index among Japanese drug-dependent patients. Int J Environ Res Public Health. 2015;12(3):2781-92. DOI: 10.3390/ijerph120302781 PMID: 25741639

25. Klika JB, Herrenkohl TI, Lee JO. School factors as moderators of the relationship between physical child abuse and pathways of antisocial behavior. J Interpers Violence. 2013;28(4):852-67. DOI: 10.1177/0886260512455865 PMID: 22929340

26. Karimi Jozestani L, Abedini M, Malekpoor M, Sadeghi A, Asli Azad M. [A comparative study of categories of positive psychologyin parents of autistic versus normal children]. JPEN. 2015;2(1):30-40. 
27. Nelson W, Finch A. Children's Inventory of Anger: ChIA Manual: Western Psychological Services; 2000.

28. Kimiaei A, Raftar M, Soltanifar A. [Efficacy based on emotional intelligence to control aggression in aggressive teenagers]. Studies Educ Psychol. 2011;1(1):153-66.

29. Spence SH. A measure of anxiety symptoms among children. Behav Res Ther. 1998;36(5):545-66. DOI: 10.1016/s0005-7967(98)00034-5

30. Mosavi R, Moradi A, Farzad V, Mahdavi Harsini E. Psychometric properties of the Spence children's anxiety scale with an Iranian sample 2007 [updated 2016]. Available from: www.scaswebsite.com.

31. Harford TC, Yi HY, Grant BF. Associations between childhood abuse and interpersonal aggression and suicide attempt among U.S. adults in a national study. Child Abuse Negl. 2014;38(8):1389-98. DOI: 10.1016/j.chiabu.2014.02.011 PMID: 24656711

32. Banducci AN, Hoffman EM, Lejuez CW, Koenen KC. The impact of childhood abuse on inpatient substance users: specific links with risky sex, aggression, and emotion dysregulation. Child Abuse Negl. 2014;38(5):928-38. DOI: 10.1016/j.chiabu.2013.12.007 PMID: 24521524

33. Lee JO, Herrenkohl TI, Jung H, Skinner ML, Klika
JB. Longitudinal examination of peer and partner influences on gender-specific pathways from child abuse to adult crime. Child Abuse Negl. 2015;47:8393. DOI: 10.1016/j.chiabu.2015.07.012 PMID: 26271556

34. Crombach A, Elbert T. The benefits of aggressive traits: a study with current and former street children in Burundi. Child Abuse Negl. 2014;38(6):1041-50. DOI: 10.1016/j.chiabu.2013.12.003 PMID: 24411982

35. Maniglio R. Child sexual abuse in the etiology of anxiety disorders: a systematic review of reviews. Trauma Violence Abuse. 2013;14(2):96-112. DOI: 10.1177/1524838012470032 PMID: 23262751

36. Marwaha S, Bebbington P. Mood as a mediator of the link between child sexual abuse and psychosis. Soc Psychiatry Psychiatr Epidemiol. 2015;50(4):661-3. DOI: 10.1007/s00127-014-0966-1 PMID: 25308056

37. Brenner I, Ben-Amitay G. Sexual revictimization: the impact of attachment anxiety, accumulated trauma, and response to childhood sexual abuse disclosure. Violence Vict. 2015;30(1):49-65. PMID: 25774414

38. Cisler JM, Koster EH. Mechanisms of attentional biases towards threat in anxiety disorders: An integrative review. Clin Psychol Rev. 2010;30(2):20316. DOI: 10.1016/j.cpr.2009.11.003 PMID: 20005616 


\title{
Comparison between Aggression and Anxiety among Child Labor with and without Sexual Abuse
}

\section{Mahboubeh Khairi Hassan ${ }^{1}$, Anahita Khodabakhshi Koolaee ${ }^{2, *}$, Davoud Taghvaee $^{3}$}

${ }^{1}$ MSc, Department of Psychology, Islamic Azad University, Branch of Arak, Arak, Iran

${ }^{2}$ Assistant Professor, Department of Counseling and Psychology, Faculty of Human Science, Khatam University, Tehran, Iran

${ }^{3}$ Assistant Professor, Department of Psychology, Islamic Azad University, Branch of Arak, Arak, Iran

* Corresponding author: Anahita Khodabakhshi Koolaee, Assistant Professor, Department of Counseling and Psychology, Faculty of Human Science, Khatam University, Tehran, Iran. E-mail: a.khodabakhshid@khatam.ac.ir

Received: 02 Jun 2016

Accepted: 26 Aug 2016

\begin{abstract}
Introduction: Child labor is one of the major social problems. However, this phenomenon is associated with sexual child abuse, which has an inevitable consequence on mental health. Therefore, the aim of current study was to compare aggression and anxiety in child labor with and without sexual child abuse.

Methods: This study was a descriptive comparative research. Sixty children with sexual child abuse and 60 without sexual child abuse were selected through convenience sampling method in Tehran, Iran in 2016. The measuring instruments were Children's Inventory of Anger (ChIA, 2000) and the Spence Children's Anxiety Scale (SCAS, 1998). Data was analyzed utilizing multivariate analysis of variance (MANOVA).

Results: The results indicated that there was a significant difference between anxiety ( $\mathrm{F}=14.427$, $\mathrm{P}=0.0001)$ and aggression $(\mathrm{F}=16.248, \mathrm{P}=0.0001)$ in child labor with and without sexual child abuse $(\mathrm{P}<0.001)$.

Conclusions: These findings emphasize that sexual child abuse should be considered as one of the several risk factors to increase anxiety and aggression, which can also intensify the level of anxiety and aggression among the child labor community.

Keywords: Aggression, Anxiety, Child Labor, Sexual Child Abuse
\end{abstract}

